\title{
On the post main sequence expansion of stars with contracting helium cores
}

\author{
M. Schrinner and W. Deinzer \\ Universitäts-Sternwarte Göttingen, Geismarlandstrasse 11, 37083 Göttingen, Germany
}

Received 12 July 2001 / Accepted 10 September 2001

\begin{abstract}
The post main sequence expansion of a $5 M_{\odot}$-star is investigated by means of a simple composite configuration: a contracting He-core of Schoenberg-Chandrasekhar mass surrounded by an H-envelope of polytropic index $n=3$. While the structure of the envelope is immediately obtained by solving the equations of hydrostatic equilibrium, the core requires some further simplification: if the actual non-local gravitational energy release due to contraction is replaced by its constant core-average, the equation of radiative energy transport may be easily integrated. Thus an explicit relation between pressure and temperature is obtained and the equations of hydrostatic equilibrium may be solved. Specifying $M, M_{\text {core }}$ and $T_{0}$ (the temperature of the H-burning shell-source at the interface), a sequence of models follows with $L_{\text {core }}$, the gravitational energy released from the core per second, and hence with $t$, the contraction time, as the parameter. The resulting simple models show very rapid expansion, a consequence of the thermostatic action of the shell-source. Its fixed temperature prevents the shell-source from participating in the contraction of the core - thus causing the outer parts of the core and hence the adjoining envelope to decrease in density. Accordingly, the envelope must expand. This consequence of a fixed temperature $T_{0}$ is clearly demonstrated by the distributions of the specific internal and gravitational energies. This characteristic behaviour is also found in stellar models obtained by elaborate numerical simulations.
\end{abstract}

Key words. stars: Hertzsprung-Russell diagram - stars: interiors - stars: evolution

\section{Introduction}

As is well-known from numerical simulations, main sequence stars expand by a large amount after having used up their central hydrogen content by nuclear burning. At this evolutionary state, a helium core forms and nuclear energy production is taking place within a thin shell around that core. What does this structural change of the deep interior of a star have to do with the vast expansion of its exterior?

It is obviously very difficult to find a clear-cut answer by studying numerical simulations of stellar evolution - see the recent paper by Sugimoto \& Fujimoto (2000). Hence, in a preceding paper (Deinzer 1999, henceforth quoted as Paper I) the following answer was deduced from a simple hydrostatic model: as soon as the isothermal helium core approaches the Schoenberg-Chandrasekhar mass, it starts to contract. Thus, its gravitational pull upon the outer part of the star (its "envelope") increases. In the case of uniform contraction of the whole core, the temperature has to increase as well, in order to balance the increasing gravity force. Yet, at the nuclear shell source an

Send offprint requests to: W. Deinzer,

e-mail: deinzer@uni-sw.gwdg.de almost constant temperature is required due to the high temperature sensitivity of nuclear energy generation rates (thermostatic action of the nuclear burning). Hence, a discrepancy arises which can only be avoided by non-uniform contraction. In fact, the simple hydrostatic model investigated in Paper I shows that the central part of the core contracts much more than its outer part. Consequently for the simple reason of mass conservation - the outer part of the core becomes less dense than it was before contraction. Because of pressure equilibrium at the shell source interface, the envelope, too, must become less dense; this means the envelope must expand.

Why should a core contract in this non-uniform manner? For the low mass stars investigated in Paper I, nonuniform contraction is caused by degeneracy: the highly contracted part of the core is completely degenerate and its structure is determined by a different equation of state than its outer non-degenerate part. In fact, as soon as degeneracy becomes important, rapid contraction is halted and the core becomes isothermal again. Thus, further evolution is independant of the preceding (time-dependant) contraction phase.

But what goes on in stars of higher mass whose cores never become degenerate? This is the topic of the present 
paper. Here contraction continues and we have a timedependant problem which can be treated in the following simplified manner:

The gravitational energy released during contraction is transported by radiation, so there must be a non-vanishing temperature gradient

$\frac{\mathrm{d}\left(\frac{a}{3} T^{4}\right)}{\mathrm{d} P}=\frac{\kappa}{4 \pi c G} \cdot \frac{L_{r}}{M_{r}}$.

The luminosity $L_{r}$, i.e. the energy transported through a sphere of radius $r$ per second, is now determined by an energy generation rate which depends not only on pressure and temperature but also on the time-derivatives of these quantities (non-local energy source). In what follows, this explicit time-dependence is avoided by replacing the spatially varying energy generation rate by its global average taken over the whole core

$\frac{L_{r}}{M_{r}}=\frac{L_{\text {core }}}{M_{\text {core }}}$

an assumption introduced already in the pioneering work by Sandage and Schwarzschild $(1952)^{1}$. The time dependence itself follows from

$L_{\text {core }}=\frac{1}{2} \frac{\Delta \int_{0}^{M_{\text {core }}} \frac{G M_{r}}{r} \mathrm{~d} M_{r}}{\Delta t}$

where $\Delta t$ is the time elapsed between two successive evolutionary states.

According to Eq. (2) and for constant opacity due to Thompson scattering, the right-hand side of Eq. (1) is constant; thus it may be immediately integrated to yield

$\frac{a}{3} T^{4}=\frac{\kappa}{4 \pi c G} \frac{L_{\text {core }}}{M_{\text {core }}} P+C$.

Except for the constant of integration, $C$, this is a polytropic relation. $C$ is needed to make the temperature at the core/envelope interface, i.e. at low values of pressure, equal to that of hydrogen burning, $T_{0}$; hence

$C \lesssim \frac{a}{3} T_{0}^{4}$

Then Eq. (4) is not a polytropic relation, but it has polytropic asymptotes. For the high values of pressure in the central part of the core, $C$ becomes unimportant and relation (4) is that of a polytrope of index $n=3$. For the low values of pressure in the outer part of the core the pressure term becomes unimportant and relation (4) approaches the condition of isothermality, i.e. $n \rightarrow \infty$. Thus a two-fold structure of the core is obtained again, which is necessary for its non-uniform contraction as pointed out above. Particularly the extended outer part of the core is a consequence of the high value of the local polytropic index expected there.

${ }^{1}$ This assumption is in contradiction to the non-uniform contraction pointed out above, but only a few percent of the mass actually behaves non-uniformly and contributes only a negligible amount to the gravitational energy release.
In Sect. 2 we present a simplified composite model, whose contracting cores are based in Eq. (4). In Sect. 3, the results are discussed and compared with those of numerical simulations.

\section{The model}

Our model star is a composite configuration consisting of a core and an envelope. In both parts only the equations of hydrostatic equilibrium

$\frac{\mathrm{d} P}{\mathrm{~d} r}=-\frac{G M_{r}}{r^{2}} \varrho$

$\frac{\mathrm{d} M_{r}}{\mathrm{~d} r}=4 \pi r^{2} \varrho$

are to be solved. Hence a relation between pressure $P$ and density $\varrho$ is needed to close the system of equations. For the core, Eq. (4) together with the equation of state

$P=\frac{k}{\mu H} \varrho T$

is such a relation. For the envelope we adopt the polytropic relation

$P=K \varrho^{\frac{n+1}{n}}$.

The polytropic constant $K$ is a free quantity and the polytropic index $n=3$ was chosen. Although any value $0 \leq n<5$ would yield reasonable qualitative results, a polytropic envelope with $n=3$ is the most realistic choice for the radiative region above the shell source.

The solutions of core and envelope are fitted to each other at the shell source interface properly taking into account the chemical discontinuity

$X_{\text {core }}=0.0, Y_{\text {core }}=0.98, Z_{\text {core }}=0.02, \quad \mu_{\text {core }}^{-1}=0.745$
$X_{\text {env }}=0.7, \quad Y_{\text {env }}=0.28, \quad Z_{\text {env }}=0.02, \quad \mu_{\text {env }}^{-1}=1.62$.

This chemical composition was used by Langer (1999) in his evolutionary computation of a $5 M_{\odot}$ star, with which our solutions will be compared.

According to the degrees of freedom, our model is uniquely determined by specifying

$M, M_{\text {core }}, T_{0}, L_{\text {core }}$.

The total mass $M=5 M_{\odot}$ was chosen. The temperature at the interface $M_{r}=M_{\text {core }}$ is that of the hydrogen burning shell source $T_{0}=2 \times 10^{7} \mathrm{~K}$, thus satisfying energy equilibrium in a simplified way. The mass of the core, $M_{\text {core }}$, has a fixed value because the contraction time-scale is much shorter than the time-scale of outward nuclear burning. It has the value of the Schoenberg-Chandrasekhar mass for the present model: $M_{\mathrm{SC}} / M=0.065$. Hence an evolutionary sequence cannot be labelled by $M_{\text {core }}$ as in Paper I. The evolutionary parameter is now $L_{\text {core }}$, the last quantity in the above list. It is directly related to the timescale by Eq. (3).

The evolutionary sequence of our simplified models starts with the isothermal core formed after depletion of 


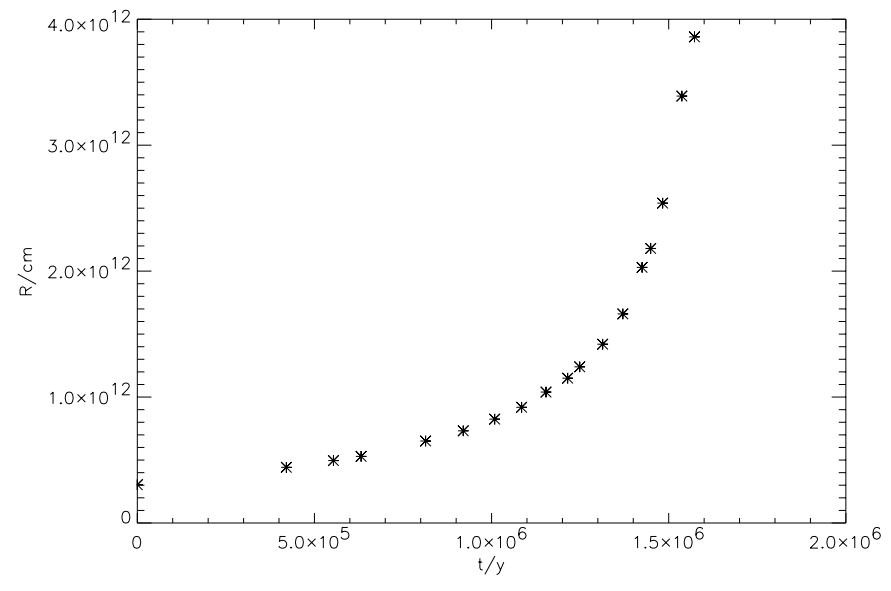

Fig. 1. Radius $R$ as a function of time $t$ for a simplified stellar model of $5 M_{\odot}$ after formation of a nuclear shell-source. At $t=0$ the isothermal core has reached its SchoenbergChandrasekhar mass and starts to contract.

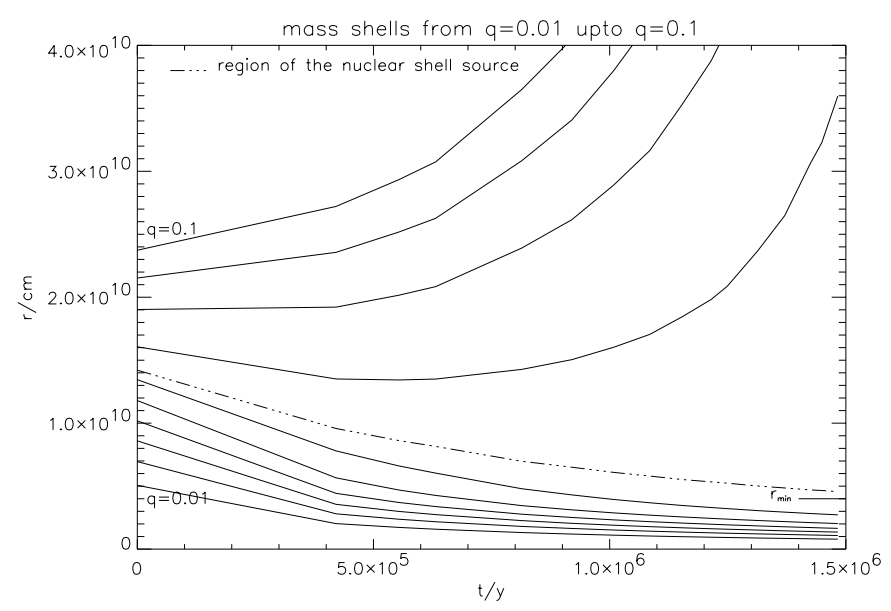

Fig. 2. The radial variation of different mass shells (labelled by the values $q=\frac{M_{r}}{M}$ from 0.01 to 0.1 ) with time demonstrating their strongly non-homologous behaviour ( $t=0$ as above).

the central hydrogen content, i.e. for $L_{\text {core }}=0$. Then contraction starts, the degree of which is characterised by increasing values of $L_{\text {core }}$. The growth of the gravitational energy per second released from the core during further evolution demonstrates the gravothermal catastrophe, into which the contracting core is evolving (and which is only stopped by the onset of helium burning). As expected, the external behaviour of these models during this evolutionary phase is indeed a strong increase of $R$ with time (Fig. 1).

Close inspection of the corresponding internal behaviour with time (Fig. 2) clearly reveals the formation of an increasingly compact inner part of the core and of an outer part of significantly smaller density.

Above the shell source interface is the strongly expanding envelope. The contraction of the interface itself is limited by

$\frac{G M_{\text {core }}}{r_{\text {core } \min }} / \frac{k T_{0}}{\mu_{\text {env }} H}=4$.

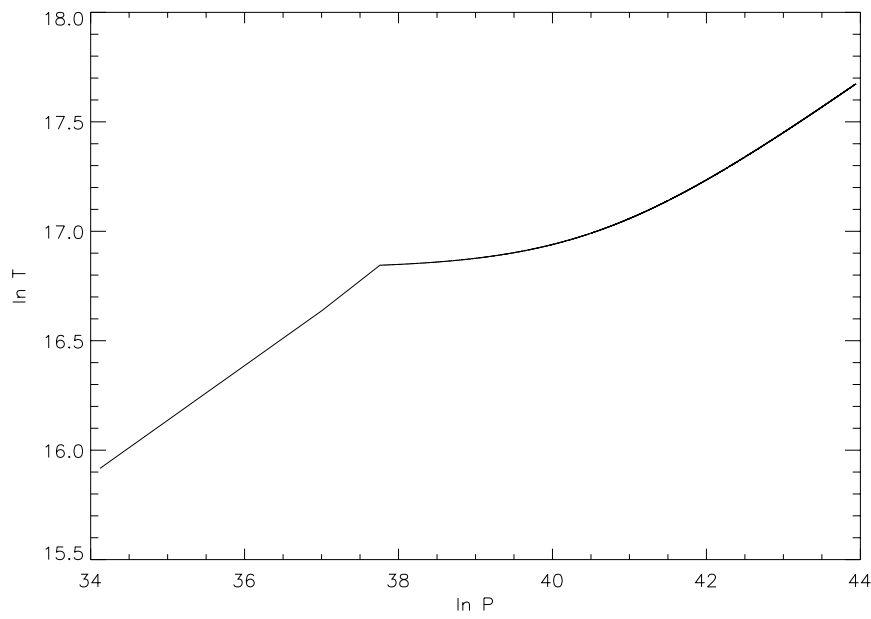

Fig. 3. The $(\ln P, \ln T)$-relation ("equation of state") for a model star at $t=1.6 \times 10^{6}$ years. Whereas the polytropic index $n=1 /\left(\frac{\mathrm{d} \ln T}{\mathrm{~d} \ln P}\right)-1$ of the envelope $(\ln P<38)$ is $n=3$, the polytropic index of the the core $(\ln P>38)$ varies from $n \rightarrow \infty$ to $n=3$ - corresponding to the non homologous contraction of the core.

This lower bound to $r_{\text {core }}$ follows from the analytical solution of the hydrostatic equation for a polytropic envelope of index $n=3$, whose mass is neglected (see appendix). Substituting $M_{\text {core }}=0.065 \cdot 5 M_{\odot}$, the SchoenbergChandrasekhar value of the mass of the core, $T_{0}=2 \times$ $10^{7} \mathrm{~K}$, the temperature of H-burning, and the chemical composition above, a lower bound for the radius of the core

$r_{\text {core }, \min }=3.9 \times 10^{9} \mathrm{~cm}$

is obtained, which is in very good agreement with the results in Fig. 2.

The two-fold structure of the core is demonstrated by the relation between temperature and pressure governing its hydrostatic equilibrium (Fig. 3); while the high pressure part is steepening, the low pressure part remains isothermal.

Our simple hydrostatic model shows the desired post main sequence expansion without invoking any sophisticated properties of stellar matter. This should be particularly emphasized because there were attempts in the past to make special properties of the opacity in the envelope responsible for its expansion. For the last word on these attempts see Iben (1993), who found expansion also for very general opacities, thus disproving this idea. Special physics will certainly modify the expansion but is not the fundamental reason for it.

\section{Discussion}

As to the the physical connection between the contracting core and the expanding envelope, our simple model points to the extended, almost isothermal layer just below the shell-source. It reduces the strong gravitational pull of the compact core upon the massive envelope which otherwise could not be balanced by the limited thermal pressure 

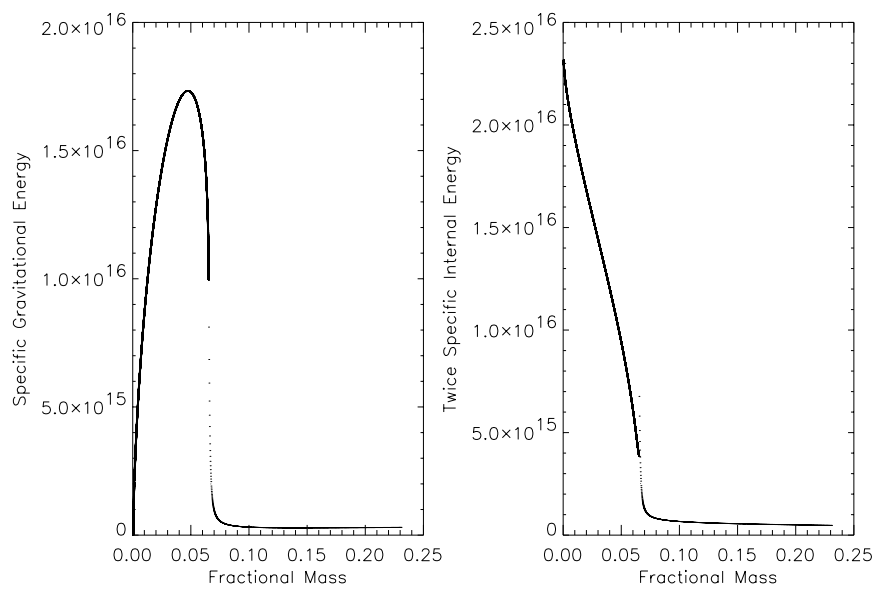

Fig. 4. Specific internal and gravitational energy as functions of fractional mass $\frac{M_{r}}{M}$ for a simplified stellar model of $5 M_{\odot}$ at $t=1.6 \times 10^{6}$ years.

due to the fixed temperature of the shell-source. It is of low density and can - for hydrostatic reasons - only be adjoined by an envelope of correspondingly low density, i.e. by an extended envelope.

These circumstances are most clearly demonstrated by the specific gravitational energy $\frac{G M_{r}}{r}$ and the specific thermal energy $\frac{k T}{\mu H}$ as functions of $M_{r}$ (Fig. 4): the latter decreases from high values at the center of order $10^{17}$ to the value $1.24 \times 10^{15}$ at $M_{r}=M_{\text {core }}$, where the fixed temperature $T_{0}=2 \times 10^{7} \mathrm{~K}$ of the shell-source is prescribed; the former is a strongly increasing function of $M_{r}$, whose steep rise is abruptly reverted shortly before reaching $M_{r}=M_{\text {core }}$.

This behaviour is a consequence of the virial theorem, which requires about equal areas below both curves. Since there is a fixed low value of the specific thermal energy at $M_{r}=M_{\text {core }}$ the specific gravitational energy must drop steeply at $M_{r} \approx M_{\text {core }}$ to match. This steep slope is identical with that of the intermediate layer. The strong decrease of $\frac{G M_{r}}{r}$ over a small mass interval implies that small densities are characteristic for this layer. The adjoining envelope is very extended as expected. Its contribution to the specific energies is almost negligible.

If the formation of the intermediate layer is decisive for post main sequence stars with extended envelopes, its signature must also be visible in the run of specific energies of stellar models obtained by numerical simulations. Such computations have been carried out by Langer (1999) and the results for a selected evolutionary state of a $5 \mathrm{M}_{\odot}$ star are presented in Fig. 5. Clearly, the steep slope of $\frac{G M_{r}}{r}$ at $M_{r} \lesssim M_{\text {core }}$ is quite conspicuous. Slight differences in $M_{\text {core }}$ are due to the Schoenberg-Chandrasekhar mass, whose exact value depends on the detailed structure of the envelope; in our models this was just a $n=3$ polytrope.

So far our models were constructed using a fixed temperature $T_{0}=2 \times 10^{7} \mathrm{~K}$ at the shell-source. In this way extended envelopes resulted regardless of the energy needed
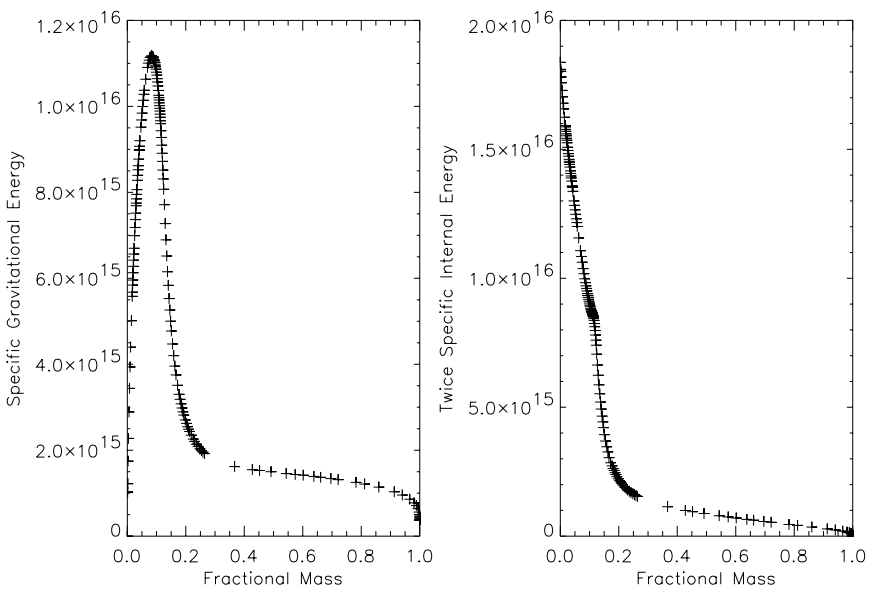

Fig. 5. Specific internal and gravitational energy as functions of fractional mass $\frac{M_{r}}{M}$ for an evolved star of $5 M_{\odot}$ obtained by numerical simulation (Langer 1999).

to produce them. To see where this energy comes from, the thermostatic action must be followed in detail. If the condition of fixed $T_{0}$ is removed, $T_{0}$ increases during contraction of the core. Thus, the shell-source would produce more energy than can be transported by the envelope. The surplus energy is used to expand the envelope, its overall density decreases, the density in the lower vicinity of the shell source and hence the temperature are reduced until their appropriate values are restituted.

\section{Conclusion}

Physically, the reason for the expansion of the present models is the same as it was for low mass stars: the contraction of the core at a fixed temperature on top of $M_{\text {core. }}$. Satisfying these two conditions requires a strongly nonuniform contraction, which essentially yields a low density outer core and an extended envelope. This fast contraction keeps on going in the present models whereas, for the low mass stars of Paper I, it is stopped by the onset of degeneracy; further evolution proceeds on a nuclear time-scale. The evolution of the present models is only prevented from gravothermal catastrophe by ignition of the central helium fuel. All energy radiated by the surface is then produced by central helium burning. The shell source becomes irrelevant and the structure of the whole star is that of a point source model. Hence the radius must shrink and the evolutionary path in the Hertzsprung-Russell-diagram performs a loop, well-known from numerical simulations (see for instance Kippenhahn \& Weigert 1994).

Acknowledgements. We are grateful to Norbert Langer for making available to us his numerical simulations of the advanced evolution of a $5 M_{\odot}$-star and to Rick Hessman for carefully checking our English manuscript.

\section{Appendix A: Derivation of Eq. (10)}

Replacing $M_{r}$ by $M_{\text {core }}$ in Eq. (6) - thus neglecting the mass of the envelope - and applying the polytropic 
relation (9), a differential equation is obtained which may be integrated immediately to yield

$K(n+1) \varrho^{\frac{1}{n}}=G M_{\text {core }}\left(\frac{1}{r}-\frac{1}{R}\right)$

where the constant of integration has been chosen to satisfy the outer boundary condition

$r=R, \quad \varrho=0$.

With the help of Eqs. (8) and (9), the left-hand side can be transformed into an expression of $T$. If the resulting equation is applied to the interface,

$(n+1) \frac{k T_{0}}{\mu_{\text {env }} H}=G M_{\text {core }}\left(\frac{1}{r_{\text {core }}}-\frac{1}{R}\right)$ is obtained, where $T_{0}$ is the temperature of H-burning. For $n=3$ and in the limit $R \rightarrow \infty$ Eq. (10) is obtained. That it is a lower bound to $r_{\text {core }}$ (and not an upper one as suggested by the above equation) is due to the nonvanishing mass of a still finite envelope.

\section{References}

Deinzer, W. 1999, A\&A, 342, 704

Iben, I., Jr. 1993, ApJ, 415, 767

Kippenhahn, R., \& Weigert, A. 1994, in Stellar Structure and Evolution (Springer, Berlin Heidelberg), 298

Langer, N. 1999, private communication

Sandage, A., \& Schwarzschild, M. 1952, ApJ, 116, 463

Sugimoto, D., \& Fujimoto, M. J. 2000, ApJ, 538, 837 